DEVELOPMENTAL BIOLOGY

\title{
On making a commitment
}

The regular tiling of the Drosophila adult eye - a precise array of $\sim 800$ units, or ommatidia — is a familiar sight to developmental biologists, who have used this organ to investigate how neuronal determination and differentiation occurs. Anatomical studies, and more recently, molecular genetic ones, have established that the eight photoreceptor cells (PRC 1-8) in each ommatidium arise through a series of stereotypical inductive interactions that take place in the larva. What isn't clear, however, is when each PRC makes the irreversible commitment to a specific fate. It is commonly thought that such a commitment is made in the larva, when each PRC expresses a distinct set of molecular markers; but Mollereau, Domínguez and colleagues now show that PRC determination occurs in two steps, each under separate genetic control. They do so by identifying the spalt ( sal) gene complex as one component of this control.

The eight types of PRC are recognizable not only by their molecular markers, but also by their distinct position within the ommatidium, by their morphology and by the types of projection that they make to the optic lobe. PRCs 1-6 - the outer photoreceptors - have larger lighttrapping organelles (rhabdomeres) and make axonal projections to the lamina; by contrast, the smaller, inner PRCs 7 and 8 project deeper in zinc-finger proteins encoded by the

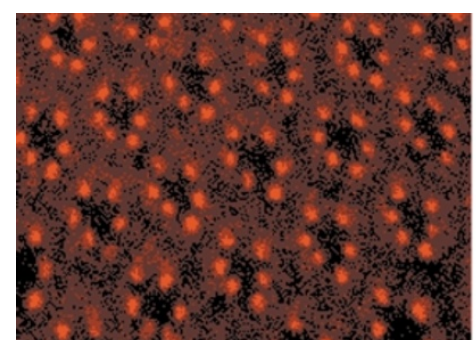
the optic lobe, to the medulla. The

two genes of the sal gene complex, sal major (salm) and sal-related (salr), have similar expression patterns and functions in eye development. When the sal complex is removed from the eye (using a chromosomal deficiency), PRCs 7 and 8 of each ommatidium are cellautonomously transformed into outer photoreceptors. However, the axons of the transformed PRC 7 and 8 cells project normally to the medulla, indicating that $s a l$ is not required for the initial inner PRC specification. Such a function for the sal-encoded proteins is consistent with their expression pattern: although salm is expressed in PRCs 3 and 4 in the larva, it is only present in PRCs 7 and 8 in the pupa and adult, at a time that coincides with $\mathrm{R} 7$ and 8 differentiation.

The data lead nicely to a model in which PRC development occurs in two steps: first, cells differentiate as neurons and send axonal projections to the brain; it's only in the second that they become mature PRCs and execute their differentiation programme. In such a model, the sal complex would be required for the terminal differentiation of PRCs 7 and 8, but not for the initial acquisition of neural characteristics by these cells. Tanita Casci

\section{(2) References and links} ORIGINAL RESEARCH PAPER Mollereau, B. et al. Two-step process for photoreceptor formation in Drosophila. Nature 412, 911-913 (2001) WEB SITE

Claude Desplan's lab:

http://homepages.nyu.edu/ cd38/

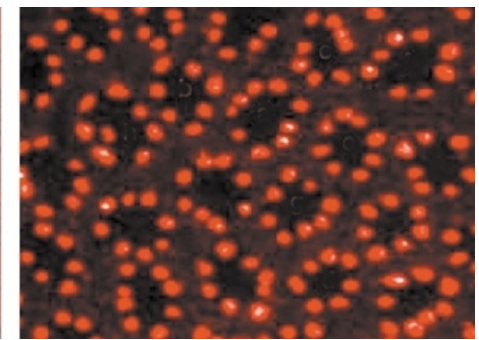

In an adult retina mutant for the spalt complex (right), all photoreceptor cells (PRCs) express the outerPRC-specific rhodopsin gene. Left, wild-type. Photo provided by Bertrand Mollereau, The Rockefeller University, New York, USA. Reproduced with permission from Mollereau, B. et al. Nature $\odot$ (2001) Macmillan Magazines Ltd.

\section{IN BRIEF}

\section{EVOLUTION}

Molecular evidence for the early colonisation of land by fungi and plants.

Heckman, D. S. et al. Science 293, 1129-1133 (2001)

Fossil evidence indicates that the establishment of eukaryotes on land occurred around 480-460 million years (Myr) ago. Molecular clock data, based on a ribosomal gene, however, place this event at around $600 \mathrm{Myr}$ ago. Now, an estimate of fungal divergence times based on the alignment of nuclear-encoded proteins pushes the origin of land eukaryotes to as early as 1,000 Myr ago.

\section{BIOINFORMATICS}

Assembly of the working draft of the human genome with GigAssembler.

Kent, W. J. \& Haussler, D. Genome Res. 11, 1541-1548 (2001)

The draft human genome sequence data have been assembled using several approaches. One of the most successful and widely used assemblies is the "Golden Path" (http://genome.ucsc.edu) pioneered by these authors. This paper provides an overview of the algorithm that underlies the Golden Path assembly, shows how genome sequence data were combined with other types of data and gives useful insight into the strengths and weaknesses of the assembly.

\section{PLANT GENETICS}

Plant growth homeostasis is controlled by the Arabidopsis BON1 and BAP1 genes.

Hua, J. et al. Genes Dev. 15, 2263-2272 (2001)

Organisms maintain their morphology under varying environmental conditions as a result of a poorly understood interplay between extrinsic and intrinsic factors. Hua et al. here identify a cell-membrane-associated, phospholipid-binding protein, $\mathrm{BON} 1$, and its partner BAP1 as factors that are necessary for normal Arabidopsis growth at low temperatures. BON1 and BAP1 co-ordinately regulate the rate of cell growth and division, possibly through their involvement in exocytosis or by controlling membrane lipid content.

\section{HUMAN GENETICS}

Human mtDNA and Y-chromosome variation is correlated with matrilocal versus patrilocal residence.

Oota, H. et al. Nature Genet. 29, 20-21 (2001)

Mitochondrial DNA often shows greater genetic diversity between human populations than does the $\mathrm{Y}$ chromosome, possibly because many human societies practice patrilocality, in which males remain at their birthplace but women migrate to join marriage partners. By collecting and analysing DNA from patrilocal and matrilocal tribes in Thailand, Oota et al. found that genetic variation does indeed strikingly correlate with patterns of residence, proving that social structure can influence human genetic diversity. 